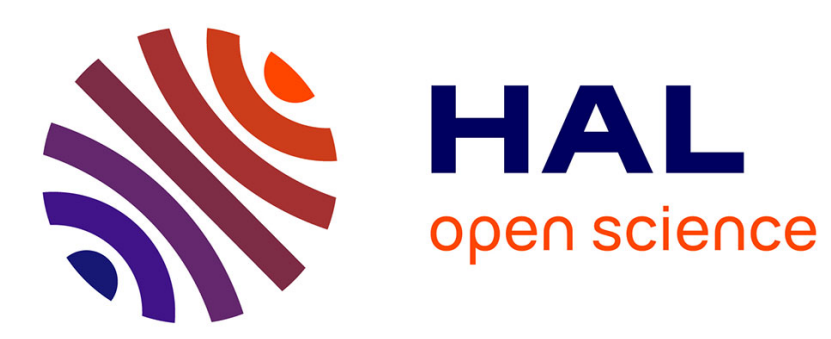

\title{
Improved light extraction with nano-particles offering directional radiation diagrams
}

Anthony Jouanin, Jean-Paul Hugonin, Mondher Besbes, Philippe Lalanne

\section{To cite this version:}

Anthony Jouanin, Jean-Paul Hugonin, Mondher Besbes, Philippe Lalanne. Improved light extraction with nano-particles offering directional radiation diagrams. Applied Physics Letters, 2014, 104 (2), pp.021119. 10.1063/1.4862189 . hal-00993242

HAL Id: hal-00993242

https://hal-iogs.archives-ouvertes.fr/hal-00993242

Submitted on 21 May 2014

HAL is a multi-disciplinary open access archive for the deposit and dissemination of scientific research documents, whether they are published or not. The documents may come from teaching and research institutions in France or abroad, or from public or private research centers.
L'archive ouverte pluridisciplinaire HAL, est destinée au dépôt et à la diffusion de documents scientifiques de niveau recherche, publiés ou non, émanant des établissements d'enseignement et de recherche français ou étrangers, des laboratoires publics ou privés. 


\title{
Improved light extraction with nano-particles offering directional radiation diagrams
}

\author{
A. Jouanin, ${ }^{1,2, a)}$ J. P. Hugonin, ${ }^{1}$ M. Besbes, ${ }^{1}$ and P. Lalanne ${ }^{1,3}$ \\ ${ }^{1}$ Laboratoire Charles Fabry, UMR 8501, Institut d'Optique, CNRS, Univ. Paris Sud 11, 2, Avenue Augustin \\ Fresnel 91127 Palaiseau Cedex, France \\ ${ }^{2}$ Saint-Gobain Recherche, Quai Lucien Lefranc, 93303 Aubervilliers, France \\ ${ }^{3}$ Laboratoire Photonique Numérique et Nanosciences, Institut d'Optique d'Aquitaine, Université Bordeaux, \\ CNRS, 33405 Talence, France
}

(Received 20 September 2013; accepted 24 December 2013; published online 16 January 2014)

\begin{abstract}
We propose a unique approach for light extraction, using engineered nano-particles to efficiently decouple the light guided in transverse-magnetic guided modes into free-space radiation modes that leak out normally to the thin-film stacks. The underlying mechanism takes advantage of a small electric field variation at the nano-particle scale and induces a "polarization conversion," which renders the induced dipole moment perpendicular to the polarization of the incident light. Our analysis is supported by 2D fully vectorial computational results. Potential applications for light emitting or photovoltaic devices are outlined. (C) 2014 AIP Publishing LLC.
\end{abstract}

[http://dx.doi.org/10.1063/1.4862189]

The realization of devices able to efficiently couple or decouple light from free space to electromagnetic guided modes impacts many technologies such as integrated optical circuits, sensing, lighting, and energy management. For instance, for light emitting devices, a significant part of the emitted light remains trapped because of the presence of guided modes and then absorbed if the film flatness is not broken. In thin-film-based solar cells, the reciprocal situation occurs, and coupling of incident photons to guided modes (also called light trapping) increases the absorption efficiency. For films with small thicknesses $(\sim 100 \mathrm{~nm})$, classical extraction solutions based on surface patterning or on embedding micron-size inclusions are not suited. Indeed, non-planar surfaces suffer from usual problems associated with material adhesion, thermal mismatch, and electrical short-cuts. For that purpose, nano-scatterers with deep subwavelength dimensions are preferred, but this approach suffers from a relatively low efficiency, since the scattering scales with the square of the scatterer volume. To increase the efficiency, metallic nano-particles (NPs) that exploit collective electron oscillation resonances are a promising route. They provide large scattering cross-sections, up to 10 times the NP size at their dipolar resonance. The latter occurs in the visible range and may be red-shifted by increasing the dielectric constant of the host material. ${ }^{1}$ Further tuning up to the near infra-red is even made possible with metallodielectric core-shell compounds. ${ }^{1,2}$ An exhaustive review on the resonances of metal NPs can be found in Ref. 3.

There are many experimental evidences that light scattering from metal NP arrays increases the performance of thin-film solar cells. ${ }^{4,5}$ In comparison, the use of individual NPs to improve the extraction efficiency of light emitting devices is much less studied and most of the underlying physical effects remain to be explored for future applications. To simplify, two effects may be exploited. ${ }^{6}$ If the NP

\footnotetext{
${ }^{\text {a) }}$ Author to whom correspondence should be addressed. Electronic mail: anthony.jouanin@institutoptique.fr
}

is located in the vicinity of the quantum emitter, near-field coupling may directly impact the emitted light, the NP acts then as an antenna. We do not intend to investigate this mechanism hereafter, but rather we focus our attention on the extraction of light trapped into guided modes. Indeed, if the NP is located far enough, one may consider that part of the emitted photons is first coupled into guided modes, before further interacting with the NP. The interaction may lead to extraction, absorption, reflexion, or even conversion into other guided modes. Through reciprocity, some of our results are also applicable for light trapping.

Very small NPs usually behave like electric dipoles at visible frequencies, with an induced dipole moment parallel to the incident electric, i.e., the polarizability tensor is diagonal. Embedded into a dielectric slab waveguide and illuminated by a transverse magnetic (TM) guided mode (such as a surface-plasmon mode in the presence of a metallic electrode), the NP dominantly acquires a dipole moment normal to the stack. This is a misfortune for extraction, since most of the radiated energy remains trapped by total internal reflection. For best performance, NPs should redirect light by acquiring a dipole moment parallel to the interfaces. Thus, a "polarization conversion" between the incident electric field $\mathbf{E}_{\mathbf{i n c}}$ and the induced dipole moment $\mathbf{p}$ has to be achieved, as depicted in Fig. 1. This requires to design NPs with a mesoscopic dimension so that the classical electricdipole behavior of deep sub-wavelength NPs is overcome. Engineering NP shapes for energy management has recently emerged, but until now most efforts focussed on light trapping in thin semiconducting layers. ${ }^{5,7}$ With the exception of the works in Refs. 8 and 9, we are not aware of any NP design for scattering light in a direction dependent on the NP orientation. In Ref. 9, this is achieved with cup-like gold NPs, yet the capability of the cup to redirect light at $90^{\circ}$ or to extract a guided mode is not documented. Similarly, the possibility of achieving directive and efficient light extraction through polarization conversion, as we show hereafter, is a design recipe not studied in the previous works. 

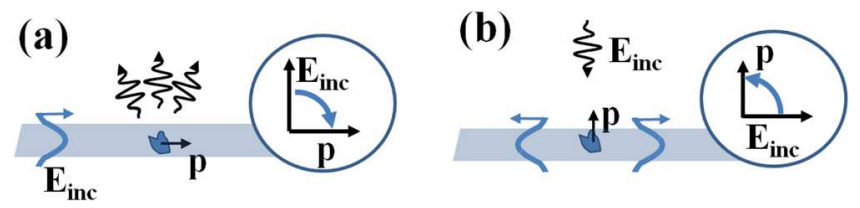

FIG. 1. Polarization conversion for coupling-decoupling free-space normally radiated waves and TM guided modes with a NP. (a) Decoupling: an incident TM guided mode is scattered into free space radiation by a NP. For best performance, the induced dipole should be parallel to the interfaces, i.e., perpendicular to the dominant electrical component of the incident field. (b) Coupling: a normally incident wave with a polarization parallel to the interface is scattered into a TM guided mode. Again as shown in the inset, the polarization of the induced dipole should be perpendicular to the incident electric field for best performance.

In this paper, we show that NP shapes can be tailored to implement polarization conversion. We unveil the excitation mechanism of the NP, and then analyze the extraction properties, assuming that the NP is embedded in a symmetric slab waveguide illuminated by the fundamental $\mathrm{TM}_{0}$ guided mode. The analysis is performed in two-dimensions- the geometry being invariant along the direction transverse to the plane of Fig. 1. Note that in 3D, polarization conversion is also required for extracting the light from TM guided modes for which, unlike TE modes, the induced dipole is dominantly perpendicular to the stack. Our goal is not to achieve a quantitative evaluation of $3 \mathrm{D}$ real situation (a task that depends on the actual thin film stack and that is much more demanding in terms of computational resources), but rather to derive design guidelines and recipes and to obtain an estimation of the capabilities and performance of the approach. Throughout the work, the numerical results are all obtained with a fully vectorial frequency-domain aperiodic-Fourier Modal method (a-FMM), ${ }^{10,11}$ and the chromatic silver metal permittivity is taken from Ref. 12 .

The achievement of polarization conversion with subwavelength nano-particles needs to overcome the classical "dipole behavior" of asymptotically small NPs. For the sake of illustration, let us start by the study of an isotropic "O" shape NP in air whose dimensions (see details in the caption of Figure 2) are adjusted such that its dipole resonance occurs at near infrared. Figure 2(a) shows the scattering efficiency $\mathrm{Q}_{\text {scatt }}$ as a function of the wavelength for several incidence angles $\theta_{\text {inc }}$ of the illuminating plane-wave. The dipole resonance at $785 \mathrm{~nm}$ offers a scattering efficiency $\mathrm{Q}_{\text {scatt }}$ close
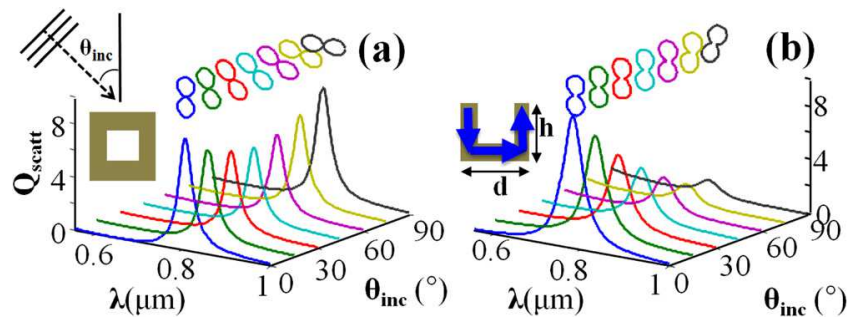

FIG. 2. Scattering efficiencies and normalized radiation diagrams of "O" and "U" NPs, illuminated by a TM-polarized plane-wave incident from air for various angles of incidence $\theta_{\text {inc }}$. The upper insets represent the normalized radiation diagrams at the resonance wavelength. (a) "O" NP composed of a square dielectric $(n=1.5)$ core covered with a 5-nm-thick silver shell. The core size is $60 \mathrm{~nm} \times 60 \mathrm{~nm}$. (b) Silver "U"-NP made in air. The blue arrows sketch the microscopic current of the resonance mode. The dimensions are $h=60 \mathrm{~nm}, d=70 \mathrm{~nm}$ and the wire thickness is $10 \mathrm{~nm}$. to 8 , almost independently of the incident beam direction. Although dipolar resonance is of great importance for many applications, the "O"- induced dipole moment rotates with the angle of incidence of the illuminating beam, as evidenced by the radiation diagrams plotted just above every spectra. This behavior is typical of isotropic (or weakly anisotropic) NPs and cannot provide polarization conversion. To take up the challenge, we consider strongly anisotropic "U"-shape NPs, whose fundamental resonance arises from a circulation of the induced current along the shape, as sketched by the blue arrows in the inset of Figure 2(b). The "U" dimensions (see details in the caption of Fig. 2) have been optimized to present a resonance frequency close to that of the "O" with the same scattering efficiency for $\theta_{\text {inc }}=0$. But, in contrast to the "O," the normalized angular diagram of the "U" (Fig. 2(b)) remains almost unchanged as $\theta_{\text {inc }}$ varies from $0^{\circ}$ to $90^{\circ}$. Indeed, as $\theta_{\text {inc }}$ tends towards $90^{\circ}$, the scattering efficiency lowers from 8 to 1 , still a relatively large efficiency. ${ }^{13}$ The key ingredient behind the directivity feature lies in the far-field radiation of the two opposites vertical currents, which almost cancel due to destructive interferences. As a consequence at frequency close to the resonant frequency, the radiation diagram dominantly results from the horizontal current induced in the base of the "U." Actually, one may note the slight tilt in the radiation diagram for $\theta_{\text {inc }}=90^{\circ}$. The latter originates from a residual contribution of a symmetric resonance at $\lambda \approx 500 \mathrm{~nm}$.

An important issue concerns the mechanism behind the excitation of the "U" resonance at $\theta_{\text {inc }}=90^{\circ}$. Unlike for normal incidence, the incident field cannot directly couple to the induced current in the horizontal wire of the "U." The coupling is provided by the vertical arms. Here, the trick lies in the finite size of the "U"-NP. Despite the tiny scale $\sim \lambda / 10$ of the NP, the field experienced by the two arms is slightly out of phase. The phase difference $\exp (\mathrm{i} k d)$ with $k$ being the free-space wave number is sufficient to excite the odd-symmetric resonance of the "U." Actually, it can be shown ${ }^{14}$ that the excitation efficiency is proportional to $\sim\left(k d \sin \left(\theta_{\text {inc }}\right)\right)^{2}$ for small $d$ and thus scales as $(d / \lambda)^{2}$ for $\theta_{\text {inc }}$ $\approx 90^{\circ}$, in qualitative agreement with numerical results.

As $\theta_{\text {inc }}$ increases up to $90^{\circ}$, the scattering efficiency of the resonant " $\mathrm{U}$ " drops to 1 , but the radiation diagram remains largely unchanged: polarization conversion is achieved.

"U"-NPs, a shape related to the so-called split-ring-resonator geometry, have received much attention from the metamaterial community ${ }^{15,16}$ because of their magnetic response that arises from the circulation of induced current. However, they were not studied in the perspective of tailoring the radiation diagram. As we shall see now, in guided optics, this perspective may offer opportunities for coupling-decoupling TM-polarized guided modes to free space radiations, even at the level of a single NP.

We now assume that the "U" and the "O" NPs are buried in a very simple symmetric waveguide, a glass $(n=1.5)$ slab with a thickness $w$ and with air claddings. First, the "U"- and "O"-NP dimensions are adjusted to resonate both at $800-\mathrm{nm}$ wavelength in the host material with a 1.5 refractive index. The "U" dimensions become $d=70 \mathrm{~nm}$, $h=50 \mathrm{~nm}$ for a silver wire thickness of $20 \mathrm{~nm}$. Similarly, the square size of the "O" and the shell thickness become 64 and 
$8 \mathrm{~nm}$, respectively. Then using the a-FMM, ${ }^{10,11}$ we numerically model the scattering of every NP under illumination by the fundamental $\mathrm{TM}_{0}$ guided-mode of the slab. Four important quantities are calculated: the modal reflectance $R$ and transmittance $T$ into the backward- and forward propagating $\mathrm{TM}_{0}$ modes, the total scattered efficiency $S$ into all the higher-order guided modes (if any), the NP absorption $A$ and the extraction efficiency $\beta^{\theta}$ into the claddings within a cone of opening angle $\theta, \beta^{\pi}$ denoting the fraction of light extracted in all angular directions (see the inset in Fig. 3(b)).

Figure 3 compares the performance of the " $\mathrm{U}$ " and "O" NPs, when the NPs are located right in the centre mirrorsymmetry plane of the slab. Because the z-component of the $\mathrm{TM}_{0}$ electric-field is null in this plane, the NP resonances are excited only by the transverse electric-field component of the incident mode, and the case is particularly suited to test the impact of polarization conversion for extraction. As evidenced by Figs. 3(a) and 3(b), the NPs offer radically different scattering behavior: the "O" presents a strong interaction with a high reflectance (blue area), a large absorption (red area) and with a strong scattering into higher-order guided-modes (dashed area). In contrast, the "U" weakly excites all these channels and its interaction is almost exclusively dominated by the extraction of the trapped light (green area). Because the "O" NP extracts slightly more energy than the "U" NP (approximately 30\% more on average on the slab thickness), one may hurriedly conclude that polarization conversion is not the key point for extraction. Actually, this conclusion deserves much more attention. One should consider that the extraction efficiency in Fig. 3 refers to a single scattering event. Indeed, light that is neither extracted nor absorbed remains guided in the slab and can be extracted by a second, third, etc., scattering event with nearby NPs. Following a simple radiative transfer picture that considers a single guided mode, the total extraction by an ensemble of randomly distributed NPs can be estimated as a geometric series that converges to $\beta^{\pi} /\left(\beta^{\pi}+A\right)$ in the limit of an infinite number of scattering events. The previous expression emphasizes that Ohmic losses are detrimental to extraction (a)

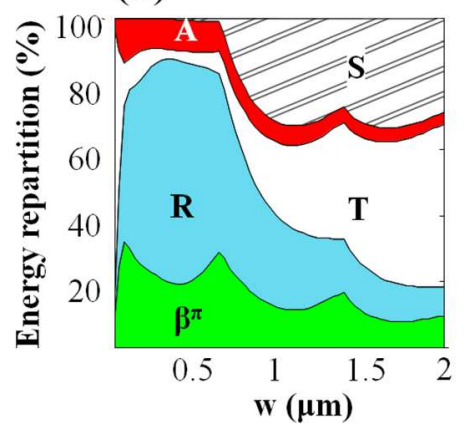

(b)

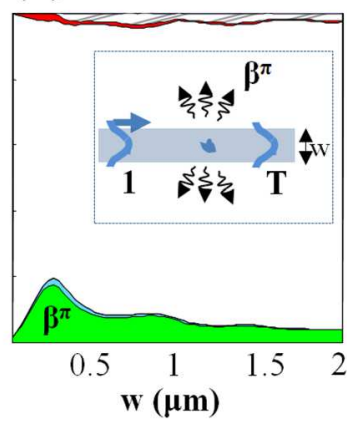

FIG. 3. Scattering properties of a single NP embedded in the centre symmetry plane of an air-glass-air slab waveguide and illuminated by the fundamental $\mathrm{TM}_{0}$ mode. The green, blue, white, red and white-hatched areas, respectively, represent the fraction of the total incident power that is extracted $\left(\beta^{\pi}\right)$, back-reflected $(R)$ or transmitted $(T)$ in the $\mathrm{TM}_{0}$ mode, absorbed $A$ by the NP, and scattered $(S)$ into higher-order waveguide modes. (a) "O"-NP with a square $64 \times 64 \mathrm{~nm}$ dielectric $(\mathrm{n}=1.5)$ core covered by an 8 -nm-thick silver shell. (b) "U"-NP with $d=70 \mathrm{~nm}, h=50 \mathrm{~nm}$ and with 20-nm-thick silver arms. The computational data are collected at the same resonance frequency $(\lambda=795 \mathrm{~nm})$ of the NPs. in a multiple-scattering-extraction scheme. It also gives more credits to the "U" for which a total extraction of $88 \%$ is predicted on average over $w$, whereas the same expression gives a smaller value of $70 \%$ for the "O." Additionally and importantly, one should also consider the directions in which the light is scattered out of the slab, a key property of the extracted radiation that is crucial for many applications.

Figure 4 displays the extraction ratio, defined as $\beta_{\mathrm{U}} / \beta_{\mathrm{O}}$, for several values of the opening angles $\theta$ (see Fig. 4(a) for a definition of $\theta$ ). The ratio is an important figure of merit for microscopy, displays or lighting applications, where light is preferentially radiated normally to the sample or device. The extraction ratio presents a contrasted oscillatory behavior as the slab thickness varies, because of the interference induced by the reflections at the interfaces of the slab. For small opening angles $\left(\theta=0^{\circ}\right.$ to $\left.10^{\circ}\right)$, the extraction ratio is remarkably large $(\approx 60)$, despite the weaker scattering efficiency of the "U"-NP. This implies that most of the power extracted by the "U"-NP is radiated out in a small opening angle normally to the slab. With increasing opening angles, the extraction ratio decreases but remains greater than 1 up to $\theta=90^{\circ}$. For $\theta=180^{\circ}$, the extraction efficiency of the "O"-NP is slightly greater than that of the "U"-NP, in agreement with the results of in Fig. 3. The red circles represent the loss ratio $A_{\mathrm{U}} / A_{\mathrm{O}}$. On average over $w$, in the extraction process, the "U"-NP absorbs approximately 7-8 times less than the "O"-NP.

We now study the capabilities of the NPs to scatter a plane-wave incident from free space into the fundamental guided mode of the slab. For that purpose, we define the trapping cross-section $\sigma_{\text {trap }}\left(\theta_{\text {inc }}\right)$ as the ratio between the electromagnetic power scattered into the backward-propagating $\mathrm{TM}_{0}$ mode and the irradiance of the plane wave that is impinging at an oblique angle $\theta_{\text {inc }}$ (cf. Fig. 5(a)). Note that trapping cross sections have units of lengths in 2D. The numerical method used for the calculation of $\sigma_{\text {trap }}\left(\theta_{\text {inc }}\right)$ relies on reciprocity arguments. It is described in Ref. 17, where the capability of isolated NPs to launch surface-plasmon-polaritons on metal surface is studied. Figure 5 presents $\sigma_{\text {trap }}\left(\theta_{\text {inc }}\right)$ for the "O" and "U"-NPs. In agreement with previous calculations, the "U"-NP offers the unique possibility to efficiently couple normally incident light. For $\theta_{\text {inc }}=0$, the cross-section is $75 \mathrm{~nm}$, a value slightly larger than the physical cross-section $d=70 \mathrm{~nm}$. This means that all the light that
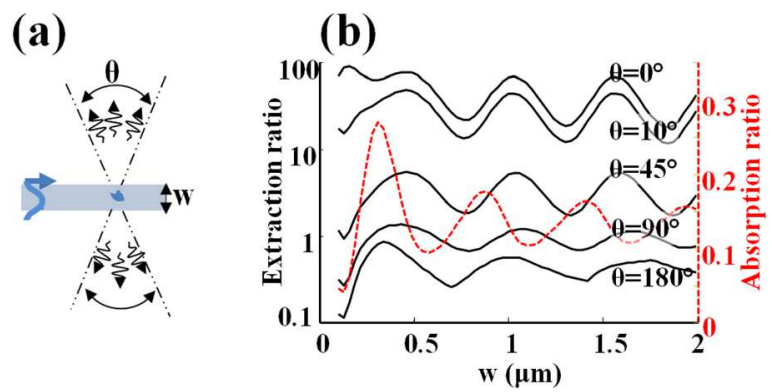

FIG. 4. (a) Definition of the opening angle $\theta$ of the radiated light. (b) Extraction ratio $\beta_{\mathrm{U}} / \beta_{\mathrm{O}}$ (black solid curves) for several opening angles $\theta$ as a function of the waveguide thickness. The dashed-red line presents the losses ratio defined as $A_{\mathrm{U}} / A_{\mathrm{O}}$. Calculations are performed at $\lambda=795 \mathrm{~nm}$ for the NP-geometries described in the caption of Fig. 3. 


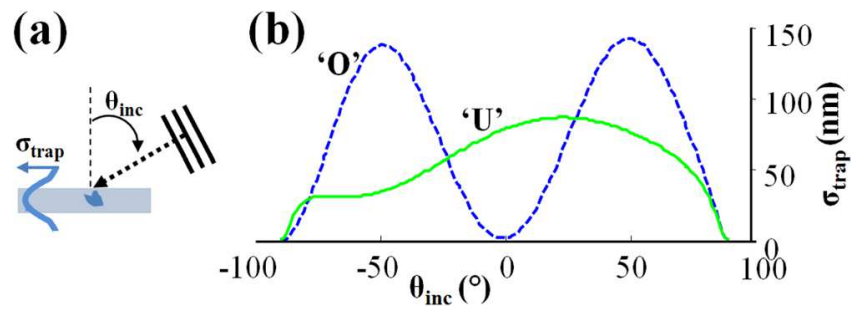

FIG. 5. (a) Sketch of the trapping of an incident TM-polarized plane-wave into the left-propagating $\mathrm{TM}_{0}$ mode of a glass slab in air. The plane wave impinges on the slab with an angle $\theta_{\text {inc }}$ and the NP is placed in the middle of the 400 nm-thick slab. (b) Trapping cross section $\sigma_{\text {trap }}$ of "U" and "O"-NPs as a function of $\theta_{\text {inc }}$. By the transformation $\theta_{\text {inc }}=-\theta_{\text {inc, }}$, the cross-section into the guided mode propagating into the right direction is obtained. The particle dimensions are given in the caption of Fig. 3 and calculations are performed at $\lambda=795 \mathrm{~nm}$.

is directly impinging onto the " $\mathrm{U}$ "-NP is converted into the backward-propagating $\mathrm{TM}_{0}$ mode (as much is converted into the forward mode for symmetry reason). In comparison, the coupling capability of the "O"-NP at normal incidence is almost null. Additionally, we note that the trapping cross-section of the " $U$ "-NP is largely asymmetric, in agreement with the radiation pattern of Fig. 1.

The present work suggests that it is possible to engineer specific NP shapes to improve the extraction of light from confined to free space. Design recipes for engineering the NPs with deep sub-wavelength sizes still need to be fully established and the evaluation of ultimate performance is still ahead. However, we hope that this preliminary 2D evaluation that predicts interesting perspectives will motivate future works with 3D NPs and more complex waveguide geometries. We are confident that easy-made 3D NPs may be designed for extracting trapped light in realistic situations. For instance, an axis-symmetrical $U$ is expected to offer higher extraction capabilities than our 2D preliminary proposal since it responds to all polarizations, either by polarization conversion for TM modes or by direct coupling for TE modes. Additionally, we believe that many degrees of freedom that we have not considered in the present work are available. For instance, the extraction or trapping efficiencies may be impacted by the transversal positioning of the NPs in the stack, the proximity of a metal interface in the near-field of the $\mathrm{NP},{ }^{18}$ or the electromagnetic interaction between nearby NPs in complex systems composed of disordered NP ensembles. $^{19}$

The authors wish to thank M. Schiavoni and G. Lecamp for fruitful discussions and Saint- Gobain Recherche for financial support.

${ }^{1}$ C. F. Bohren and D. R. Huffman, Absorption and Scattering of Light by Small Particles (Wiley-VCH, 1983).

${ }^{2}$ A. L. Aden and M. Kerker, J. Appl. Phys. 22, 1242 (1951).

${ }^{3}$ J. Zhang and L. Zhang, Adv. Opt. Photon. 4, 157 (2012).

${ }^{4}$ H. A. Atwater and A. Polman, Nature Mater. 9, 205 (2010).

${ }^{5}$ V. E. Ferry, L. A. Sweatlock, D. Pacifici, and H. A. Atwater, Nano Lett. 8, 4391 (2008).

${ }^{6}$ X. Gu, T. Qiu, W. Zhang, and P. K. Chu, Nanoscale Res. Lett. 6, 199 (2011).

${ }^{7}$ K. R. Catchpole and A. Polman, Appl. Phys. Lett. 93, 191113 (2008).

${ }^{8}$ N. A. Mirin and N. J. Halas, Nano Lett. 9, 1255 (2009).

${ }^{9}$ N. S. King, Y. Li, C. Ayala-Orozco, T. Brannan, P. Nordlander, and N. J. Halas, ACS Nano 5, 7254 (2011).

${ }^{10}$ E. Silberstein, P. Lalanne, J. P. Hugonin, and Q. Cao, J. Opt. Soc. Am. A 18, 2865 (2001).

${ }^{11}$ M. Besbes, J. P. Hugonin, P. Lalanne, S. van Haver, O. T. A. Janssen, A. M. Nugrowati, M. Xu, S. F. Pereira, H. P. Urbach, A. S. van de Nes, P. Bienstman, G. Granet, A. Moreau, S. Helfert, M. Sukharev, T. Seideman, F. I. Baida, B. Guizal, and D. Van Labeke, J. Eur. Opt. Soc. Rapid. Publ. 2, 07022 (2007).

${ }^{12}$ P. B. Johnson and R. Christy, Phys. Rev. B 6, 4370 (1972).

${ }^{13}$ This value remains high compared to the dipole-resonance efficiencies of dielectric $\mathrm{TiO}_{2}\left(\mathrm{Q}_{\text {scatt }} \sim 0.01\right)$ or metallic silver $\left(\mathrm{Q}_{\text {scatt }} \sim 0.1\right)$ "O"-shape NPs with similar sizes.

${ }^{14} \mathrm{At}$ a frequency close to a resonance frequency, the excitation of a NP by an incident field is proportional to the overlap integral between the resonance mode electric field and the incident electric field, integrated on the NP volume, see details in Q. Bai, M. Perrin, C. Sauvan, J. P. Hugonin, and P. Lalanne, Opt. Express 21, 27371 (2013).

${ }^{15}$ J. Petschulat, J. Yang, C. Menzel, C. Rockstuhl, A. Chipouline, P. Lalanne, A. Tünnermann, F. Lederer, and T. Pertsch, Opt. Express 18, 14454 (2010).

${ }^{16}$ J. Zhou, T. Koschny, and C. M. Soukoulis, Opt. Express 15, 17881 (2007).

${ }^{17}$ H. Liu, P. Lalanne, X. Yang, and J. P. Hugonin, IEEE J. Sel. Top. Quantum Electron. 14, 1522 (2008).

${ }^{18}$ A. Moreau, C. Ciracì, J. Mock, R. Hill, Q. Wang, B. J Wiley, A. Chilkoti, and D. Smith, Nature 492, 86 (2012).

${ }^{19}$ K. Vynck, M. Burresi, F. Riboli, and D. S. Wiersma, Nature Mater. 11, 1017 (2012). 